\title{
Review
}

International Archives of

Allergy Immunology

\section{Pericytes in Chronic Lung Disease}

\author{
Jessica E. Rowley Jill R. Johnson \\ Leukocyte Biology Section, National Heart and Lung Institute, Imperial College London, London, UK
}

\section{Key Words}

Allergic asthma - Chronic obstructive pulmonary disorder . Fibrosis · Inflammation · Lung · Stem cells

\begin{abstract}
Pericytes are mesenchymal cells embedded within the abluminal surface of the endothelium of microvessels such as capillaries, pre-capillary arterioles, post-capillary and collecting venules, where they maintain microvascular homeostasis and participate in angiogenesis. In addition to their roles in supporting the vasculature and facilitating leukocyte extravasation, pericytes have been recently investigated as a subpopulation of mesenchymal stem cells (MSCs) due to their capacity to differentiate into numerous cell types including the classic MSC triad, i.e. osteocytes, chondrocytes and adipocytes. Other studies in models of fibrotic inflammatory disease of the lung have demonstrated a vital role of pericytes in myofibroblast activation, collagen deposition and microvascular remodelling, which are hallmark features of chronic lung diseases such as asthma, chronic obstructive pulmonary disorder, pulmonary fibrosis and pulmonary hypertension. Further studies into the mechanisms of the pericyte-to-myofibroblast transition and migration to fibrotic foci will hopefully clarify the role of these cells in chronic lung disease and confirm the importance of pericytes in human fibrotic pulmonary disease.

(c) 2014 S. Karger AG, Basel
\end{abstract}

(C) 2014 S. Karger AG, Basel

$1018-2438 / 14 / 1643-0178 \$ 39.50 / 0$

\section{Pericytes: An Overview}

Pericytes are mesenchymal cells embedded within the abluminal surface of the endothelium of microvessels such as capillaries, pre-capillary arterioles and post-capillary collecting venules, where they maintain microvascular homoeostasis and participate in angiogenesis (fig. 1a) [1-3]. The expression of desmin and $\alpha$-smooth muscle actin ( $\alpha$-SMA) points to the contractile functions of pericytes, by which they are able to control the constriction and dilation of blood vessels, thereby regulating blood flow [4]. Indeed, pericytes occupy a phenotypic continuum with vascular smooth muscle cells (VSMCs), i.e. the mural cells located on larger blood vessels, and therefore share many characteristics $[1,5]$. The basement membrane of pericytes is continuous with endothelial cells (ECs), and the two cell types form tight associations by way of adhesion plaques composed of fibronectin and peg-and-socket formations containing tight, gap and adherens junctions [1]. The secretion of platelet-derived growth factor (PDGF)-BB by ECs recruits pericytes to the microvasculature in a paracrine fashion via PDGF receptor (PDGFR)- $\beta$ [6]. Conversely, pericytes signal to the endothelium via the angiopoietin (Ang)/Tie receptor axis. The Tie 2 receptor is expressed on ECs and recognizes the agonist Ang1, secreted by pericytes, as well as the autocrine antagonist Ang2 [6]. The Ang1 signalling loop maintains vascular stability by maintaining pericyte cov-

\section{KARGER}

E-Mail karger@karger.com www.karger.com/iaa
Correspondence to: Dr. Jill R. Johnson Imperial College London Sir Alexander Fleming Building, SAF Room 369 South Kensington Campus, London SW7 2AZ (UK) E-Mail jill.johnson@imperial.ac.uk 


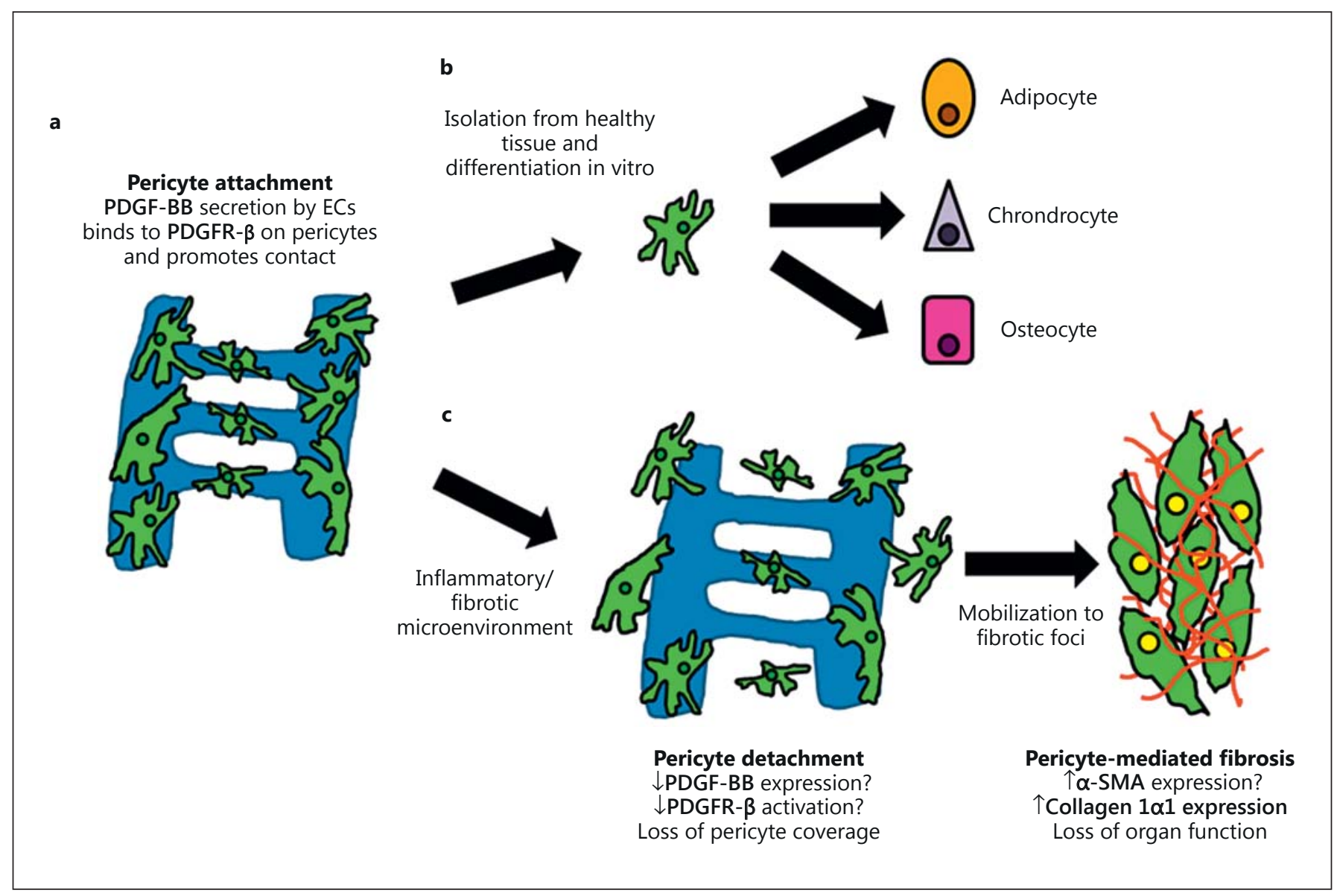

Fig. 1. a Pericytes are mural cells embedded within the abluminal surface of the endothelium of microvessels such as capillaries, precapillary arterioles, post-capillary and collecting venules, where they maintain microvascular homoeostasis and participate in angiogenesis. The secretion of PDGF-BB by ECs recruits pericytes to the microvasculature in a paracrine fashion via PDGFR- $\beta$. b Upon isolation from healthy tissue, pericytes are capable of undergoing trilineage (osteo-, adipo- and chondrogenic) differentiation similar to bone marrow-derived MSCs. c However, under inflammatory/fibrotic conditions, pericytes can migrate from the vasculature towards fibrotic foci, where they have been shown to differentiate into a myofibroblast-like phenotype. erage, whilst Ang2 overexpression is associated with pericyte loss [1]. The vessel coverage of pericytes varies according to species, organ and vessel type and ranges from $1: 1$ to $10: 1$ (ECs:pericytes) with the highest abundance in the central nervous system and retina. Sparse coverage is seen in tissues involved in gas and nutrient exchange [2, $3,7]$.

The identification of pericytes can be problematic as markers are unspecific and dynamically and differentially expressed depending on the species, type/origin of vessel and pathology [2]. Markers commonly expressed by pericytes include the tyrosine kinase receptor PDGFR- $\beta$, the chondroitin sulphate proteoglycan neural glial antigen 2 (NG2), the adhesion molecule CD146, the contrac-

Pericytes in Chronic Lung Disease tile proteins desmin and $\alpha$-SMA, the endosialin CD248, the aminopeptidase CD13, the growth factors Ang1 and Ang2, alkaline phosphatase, the GTPase signalling molecule regulator of G-protein signalling 5 and the ganglioside antigen $3 G 5[2,7,8]$. a-SMA expression is found in the more stellate pericytes of the pre- and post-capillary vessels but is lacking in spindle-shaped capillary pericytes [9]. In humans, pericytes are easily isolated from various tissues, including the lung, using fluorescence-activated cell sorting with the gating strategy CD45-/CD31-/ CD56-, CD146 ${ }^{\text {high }}[10,11]$. In mice, pericyte isolation and/or characterization has generally relied on culture methods using cells isolated from the heart [12], ear [13] and brain [14], or through the use of transgenic mice in 
which pericytes are labelled in the kidney [15], skeletal tissue [16], liver [17], lung [18-20] and brain [21]. Fluorescence-activated cell sorting is a rapid method and yields pure populations, which is not possible using culture methods alone. Following sorting, pericytes retain the expression of their characteristic markers NG2, CD146, PDGFR- $\beta$, alkaline phosphatase and $\alpha$-SMA over extended periods of culture [11].

Pericytes are thought to represent a subpopulation of mesenchymal stem cells (MSCs) due to their capacity to differentiate into numerous cell types including the classic MSC triad, i.e. osteocytes, chondrocytes and adipocytes, in addition to other cells such as fibroblasts/myofibroblasts, myointimal cells and VSMCs [2]. Initially isolated from murine bone marrow in 1966 [22], MSCs are now believed to reside in all vascularized adult tissue. Their pluripotency [23] and potential for applications in regenerative medicine and tissue engineering renders the isolation, culture and characterization of these cells desirable. In accordance with the minimal criteria proposed by the Mesenchymal and Tissue Stem Cell Committee of the International Society for Cellular Therapy, an MSC must be plastic adherent, uniformly express the MSC markers CD73, CD90 and CD105 and possess trilineage (osteo-, adipo- and chondrogenic) differentiation capabilities [3, 24]. In fulfilment of these criteria, Crisan et al. [11] showed that cultured pericytes from a range of human tissue are plastic adherent, are capable of trilineage differentiation and express CD10, CD13 and CD44. The expression of these markers persisted over extended time periods [11]. Moreover, pericytes from skeletal muscle and non-muscle tissue have been shown to be myogenic both in culture and in SCID-NOD mice injured with cardiotoxin [11] and to differentiate into myofibroblasts in models of fibrosis $[15,17,18,20]$. The landmark study by Crisan et al. [11] was the first to isolate, culture and differentiate pure populations of pericytes and provided strong evidence that they comprise an MSC-like population possessing the ability to differentiate into multiple tissue types distinct from their origin. The mesenchymal progenitor cell characteristics of pericytes have been observed in cells derived from the retina [25], endometrium [26] and skeletal muscle (fig. 1b) [27].

Although little is known about the MSC capacity of pulmonary pericytes, murine and human resident lung MSCs have been identified using the gating strategy Hoechst $33342^{\mathrm{dim}}$, CD45-, and were found to express the MSC markers ABCG2, CD90, CD105, CD106, CD73, CD44 and Sca 1 and to lack haematopoietic markers such as CD45, CD34 and c-kit [28-32]. Although pericyte marker expression on these cells was not analysed in the majority of these studies, it is likely that some of these resident lung MSCs were of a perivascular nature, as $8.7 \%$ of CD45-/CD34-/Scal + cells expressed PDGFR- $\beta$ [29].

Pericytes have been implicated in pathological processes such as fibrosis, tumour formation, hypertension and microangiopathy $[2,7]$; their role in chronic lung disease is the subject of this review. Although the presence of pulmonary pericytes was first confirmed in 1974 by electron microscopy [33], there have been a limited number of studies on pericytes in the lung, and their presence has been a somewhat controversial topic. Recent murine immunohistochemical analyses have shown desmin $+/$ NG2+/PDGFR- $\beta+/ \alpha-S M A-$ pericyte coverage of both the peribronchiolar/vascular and the alveolar regions of the lung $[18,20]$. These cells were shown to be derived from Foxd1-expressing embryonic progenitors, hinting at a mesenchymal lineage [18]. NG2DsRedBACtransgenic reporter mice have revealed an elongated, branched pulmonary pericyte structure which suggested that the expression of NG2 and 3G5 is irregular [19], again stressing the difficulties in obtaining suitable markers for pericyte investigations. This elongated structure was also shown in human lung and cultured human pulmonary pericytes isolated immunomagnetically using an anti-3G5 antibody coupled to anti-IgM microbeads [19]. In this study, $96 \%$ of the pericytes were double positive for 3G5 and NG2 [19]. The above studies confirm the presence of pericytes in both mouse and human lung, paving the way for determining their role in pulmonary pathology.

\section{The Role of Pericytes in Coordinating the Inflammatory Response}

Many chronic diseases of the lung are associated with a robust inflammatory response, in particular asthma and chronic obstructive pulmonary disorder (COPD) [34] as well as, more controversially, pulmonary fibrosis [35]. It is well known that the vascular endothelium plays a vital role in the extravasation and tissue infiltration of inflammatory cells. However, recent studies have demonstrated an equally important role of pericytes in facilitating leucocyte extravasation. Following the well-described processes of tethering and rolling on activated ECs, adhesion, crawling and transendothelial migration, leukocytes breach the vascular basement membrane and encounter pericytes that surround the blood vessel and effectively 
form an additional barrier to be crossed [36]. Gaps between pericytes are associated with regions of low expression of the basement proteins laminin and collagen type IV and high expression of ICAM-1, which essentially form portals that have been shown to be preferential sites for cell migration [37]. Recent evidence has suggested that migrating neutrophils and monocytes actively search for these regions during extravasation [38].

In vitro investigations into this process using human $\mathrm{EC} /$ pericyte cocultures have revealed that pericytes alone are less efficient at facilitating neutrophil transmigration than ECs, while EC/pericyte bilayers support intermediate levels of transmigration. This deficiency in pericytemediated neutrophil transmigration was attributed to the altered expression pattern of ICAM-1 on pericytes in the $\mathrm{EC} /$ pericyte bilayers, in which pericytes formed a confluent layer rather than providing gaps for transmigrating cells, as has been described in vivo [39]. Subsequent studies from the same research group improved on this model by incorporating a pericyte-deposited basement membrane, with ECs and pericytes seeded on opposing sides of a Transwell membrane. Interestingly, in the presence of a basement membrane, EC/pericyte composites performed comparably to ECs alone following activation with TNF- $\alpha$ [40]. Other studies have shown that pericytes can further contribute to coordinating the immune response by responding to damage-associated molecular patterns such as necrotic cell lysates or TNF- $\alpha$. The proinflammatory pericyte phenotype was characterized by upregulated ICAM-1 expression and a release of the chemoattractant MIF; using two-photon microscopy, these changes were found to greatly enhance leucocyte extravasation. Following extravasation, pericytes instructed leucocytes by increasing TLR expression and enhanced the ability of innate immune cells to scan the local microenvironment [41].

This interplay between leucocytes and pericytes during acute inflammatory responses was elegantly described by Proebstl et al. [42] using real-time confocal intravital imaging. To achieve this, chimeric mice were generated to express RFPcherry in pericytes and GFP in neutrophils. These authors showed that pericytes facilitate transmigration in vivo in an ICAM-1-, Mac-1- and LFA-1-dependent manner. The permissive extravasation sites between pericytes previously described by this research group were found to be enlarged in inflamed tissues in a process mediated by a change in the shape of pericytes that was driven by inflammatory mediators such as IL- $1 \beta$ and TNF- $\alpha$ [42]. Interestingly, only a small proportion of these gaps (9\%) were found to support the extravasation of the majority (70\%) of neutrophils; these gaps were characterized by a high expression of KC and ICAM-1 and were of a size preferred by transmigrating neutrophils, i.e. $8-50 \mu \mathrm{m}^{2}$ [42]. Mechanistically, the opening of these gaps between pericytes may depend on direct contact with neutrophils, which induces relaxation of the pericyte cytoskeleton via RhoA/ROCK inhibition [43]. Other clues to the mechanisms by which pericytes mediate leucocyte extravasation can be taken from studies on the blood-brain barrier, of which pericytes are an important component [44]. Pericytes have been shown to directly modulate inflammatory responses at the bloodbrain barrier by responding to and producing inflammatory cytokines and $\mathrm{NO}$ [45] as well as by altering their expression of integrins with a switch from $\alpha 1$ to $\alpha 2$ integrin [46].

In the respiratory tract, the role of pericytes in mediating vascular stability during inflammation has been investigated using a mouse model of Mycoplasma pulmonis infection. This study demonstrated that PDGF- $\beta$ signalling in pericytes, which is important in maintaining ECpericyte contacts, plays a vital role in maintaining vascular stability following $M$. pulmonis infection, which is characterized by an extensive vascular remodelling and increased pericyte coverage of the microvasculature in the large airways. Specifically, the inhibition of PDGF- $\beta$ signalling by the DNA aptamer AX102 in the context of acute $M$. pulmonis infection led to a greatly reduced pericyte coverage of the microvasculature of the trachea, vascular swelling and increased vascular leakiness; this was associated with an increased infectious burden in the airways determined by $M$. pulmonis 16S RNA expression [47]. It is tempting to speculate that this loss of pericytes on the respiratory microvasculature may have compromised leucocyte extravasation, thereby reducing bacterial clearance and exacerbating the disease, but further studies are needed to test this hypothesis.

\section{The Pathology and Mechanisms of Organ Fibrosis}

Fibrotic disease is a major cause of mortality throughout the world with no currently available treatment [48]. Fibrosis is the permanent scarring, thickening and hardening of tissue due to dysregulated repair mechanisms and the deposition of extracellular matrix (ECM) proteins. Typically, it occurs following chronic sustained inflammation and usually in a person with a genetic predisposition [49-51]. In the lung, fibrotic diseases, known collectively as interstitial lung disorders, manifest in 
many ways and are associated with environmental exposure (asbestos, antigens and viruses) or chronic inflammatory disease (rheumatoid arthritis and sarcoidosis) or, more commonly, are idiopathic [52]. Lung fibrosis, especially idiopathic lung fibrosis (IPF), has a poor prognosis and leads to a high mortality rate [53].

Following tissue insult, epithelial cells and ECs orchestrate the development of a temporary ECM, and cytokine release from an influx of leucocytes propels the inflammatory response and results in fibroblast recruitment [49, 53]. Lymphocytes secrete profibrotic cytokines and growth factors such as transforming growth factor (TGF) $\beta$ and PDGF [49]. Fibroblasts become activated $\alpha$-SMA+ myofibroblasts and secrete ECM proteins, particularly collagen [53]. Due to its potent effects on collagen and other ECM protein secretion, TGF- $\beta$ is thought to be a key molecule involved in fibrogenesis [50]. In the healthy lung, the temporary ECM is degraded by matrix metalloproteases, which are in turn regulated by tissue inhibitors of metalloproteases. Under conditions of chronic inflammation, however, these mechanisms are dysregulated in such a way that collagen and ECM proteins are deposited at a greater rate than at which they are degraded, resulting in fibrotic scar formation [49].

The origin of myofibroblasts, the cells considered to be the major contributors to fibrosis, remains under debate; these cells may be derived from tissue-resident cells, bone marrow-derived MSCs or cells that have undergone epithelial-to-mesenchymal or endothelial-to-mesenchymal transition [54]. In vogue, the pericyte, with its MSC-like capabilities, has been shown to significantly contribute to the myofibroblast population via lineage tracing in skin [55], muscle [55], kidney [56], liver [17, 57] and lung [18, 20] models of fibrosis. Moreover, these studies provide in vivo evidence that epithelial-to-mesenchymal transition, which until recently had been the favoured candidate, is not a major mechanism of fibrosis, whilst MSCs and resident fibroblasts play an important role.

\section{Pericytes in Organ Fibrosis}

With the advent of genetic fate-tracking techniques, it has become possible to observe changes in the localization and behaviour of pericytes in organ fibrosis. In 2008, Lin et al. [15] observed that collagen type I al (collagen 1 1 1)expressing cells (genetically labelled with eGFP) in a mouse model of renal fibrosis express markers for pericytes, including NG2 and PDGFR- $\beta$. Further analysis indicated that the number of pericytes expressing collagen
$1 \alpha 1$ increased over time following renal injury, and that these cells acquired $\alpha$-SMA expression and migrated into interstitial fibrotic areas [15]. A follow-up study from the same research group [56] used a Cre-mediated labelling strategy to demonstrate that Foxd1-expressing renal stromal cells (VSMCs, pericytes and mesangial cells) respond to renal injury by proliferating and increasing their expression of $\alpha$-SMA. In fact, the authors stated that this cell population, also positive for PDGFR- $\beta$ expression, represented nearly the entire myofibroblast population in their model of renal fibrosis [56]. Taken together with their previous studies on collagen 1al-expressing cells, these authors concluded that the vast majority of myofibroblasts in renal fibrosis are derived from pericytes, not epithelial cells, and that efforts to develop novel treatments for renal fibrosis would be best served by focusing on pericyte biology rather than epithelial-to-mesenchymal transition.

Similar outcomes have been observed in mouse models of hepatic fibrosis. Liver-resident pericytes are also known as hepatic stellate cells (HSCs); recent studies have shown that HSCs are the primary source of collagen in the liver following injury and the initiation of fibrosis. Using a combination of a reporter mouse line (eGFP-expressing collagen 1a1-producing cells) and flow cytometry to detect vitamin A-expressing HSCs in alcohol- or carbon tetrachloride-driven mouse models of liver fibrosis, it was found that the majority of myofibroblasts were derived from HSCs [57]. Further evidence to support a role for pericytes in liver fibrosis has been recently provided by lineage tracing of HSCs in three discrete models of liver disease, including models of toxic, cholestatic and fatty liver damage [17]. This lineage tracing strategy, based on Cre expression driven by lecithin-retinol acyltransferase, was found to reliably label $99 \%$ of HSCs in the healthy liver; these cells were also positive for the pericyte markers desmin and PDGFR- $\beta$. Following injury to the liver, HSCs were found to be the dominant source of myofibroblasts [17], in line with investigations into the role of pericytes in kidney fibrosis.

\section{Pericytes in IPF}

There have been only two in vivo studies investigating the role of pericytes in IPF $[18,20]$; both studies revealed heterogeneous populations of lung-resident MSCs contributing to the fibrotic phenotype and provided evidence that pericytes are major, but not sole, contributors to myofibroblast progenitor populations and collagen deposition. 
Foxd 1 is a forkhead transcription factor activated in MSC progenitors of the VSMC-pericyte lineage during embryonic development and is silenced once progenitor cells enter lung buds and differentiate into the pulmonary mesenchyme [18]. Using Foxd1-Cre and collagen 1a1-GFP transgenic mice, Hung et al. [18] identified two populations of lung-resident pericytes derived from the Foxd1 lineage and expressing the pericyte markers PDGFR- $\beta$, NG2 and CD146. One population expressed collagen $1 \alpha 1$ and was PDGFR- $\alpha-$, whilst the other was negative for both. A third lung-resident cell type was identified: collagen $1 \alpha 1+$, which did not express pericyte markers and was considered to be a classic fibroblast. Despite the pericyte-like morphology and close approximation to the microvasculature of both populations of Foxd1 progenitor-derived cells, transcriptomics revealed that collagen $1 \alpha 1-$ cells expressed genes relating to vascular development, whilst collagen $1 \alpha 1+$ cells possessed transcripts associated with matrix remodelling and wound repair-related pathways [18]. These findings may hint at an intermediate phenotype between a pericyte and a collagen $1 \alpha 1+$ non-Foxd1-derived fibroblast, in line with an MSC-like nature. In this same study, using a bleomycin-driven murine model of IPF, 45-68\% of the myofibroblasts (defined by a-SMA expression) were shown to be derived from cells of the Foxd1 lineage, and $30 \%$ of these cells were found to produce collagen within fibrotic foci. Using a similar model of bleomycin-driven lung injury in mice, Rock et al. [20] showed an increased expression of the pericyte markers PDGFR- $\beta$, NG2 and desmin within fibrotic foci. Using NG2CreER BAC-transgenic mice crossed with ROSAfarnesylated GFP reporter mice, it was shown that pericytes proliferate under fibrotic conditions, although, in contrast to the results of Hung et al. [18], few took on the $\alpha$-SMA+ phenotype of a myofibroblast. However, the number of pericytes contributing to the myofibroblast population in this study may have been underestimated due to the inefficiency of the recombination process used.

These studies provide evidence that, as in other tissues, pericytes are key cells involved in pulmonary fibrosis and contribute to a significant proportion of the myofibroblast population. However, it is clear that other cells, such as the collagen $1 \alpha 1+$ fibroblasts, which comprise $53 \%$ of the collagen-producing cells [18], are also responsible for collagen deposition. Further studies are certainly warranted to investigate whether targeting the pericyte-tomyofibroblast transition is a viable treatment strategy for IPF.

Pericytes in Chronic Lung Disease

\section{Pericytes in COPD}

$\mathrm{COPD}$ is a progressive, poorly reversible disease in which airflow is restricted as a result of peribronchial fibrosis, emphysematous changes and reduced elastic recoil. Currently, there are no studies directly investigating the role of pericytes in COPD; however, fewer a-SMA+ myofibroblasts have been shown in the alveoli and bronchioles of COPD patients compared with non-smokers, with greater numbers of $\alpha$-SMA+ myofibroblasts in the bronchi [58]. Fibroblasts also exhibit deficient contractility and migration in COPD patients [59]. These findings might suggest dysregulated pulmonary repair mechanisms involving myofibroblasts, which, as previously discussed, have been shown to be, at least in part, derived from pericytes.

Despite the fact that there has been no research into pericytes in COPD, clues to their potential role can be garnered from studies investigating the vascular response in this disorder, as pericytes participate in vascular homoeostasis and remodelling, and respond to and secrete vascular growth factors. Although little is known about microvasculature remodelling in COPD, a slightly increased vascularity has been observed $[60,61]$. An increased expression and gene polymorphisms of TGF- $\beta_{1}$ are observed in COPD [62], and this growth factor is considered a major mediator of COPD due to its proliferative effect on fibroblasts [63]. However, the TGF- $\beta_{1}$ signalling pathway is also involved in the control of the contractility of retinal pericytes [64], and therefore could mediate the vessel dilation observed in COPD. Furthermore, a smoking-induced impairment of vasodilator responses persists in COPD patients despite smoking cessation [65]. These studies suggest that dysregulated pericyte contractile activity may play a part in COPD pathogenesis.

An increased expression of vascular endothelial growth factor (VEGF), which has vasculo- and angiogenic properties, has been observed in chronic bronchitis, an aspect of COPD pathology in some patients. Moreover, VEGF levels were found to negatively correlate with forced expiratory volume in $1 \mathrm{~s}\left(\mathrm{FEV}_{1}\right)$ [66]. Of note, VEGF was increased in the VSMCs of the bronchioles and alveoli [66]. In patients with emphysema, however, VEGF levels were decreased and positively correlated with $\mathrm{FEV}_{1}$, suggesting different mechanisms with regard to the microvasculature between different phenotypes of COPD [66, 67]. VEGF may contribute to emphysema by inducing EC apoptosis [68], which could contribute to the microvessel loss observed in emphysematous COPD subtypes. Pericytes secrete VEGF $[2,69]$ and play a strong role in an- 
giogenesis and vascular homoeostasis, so it is reasonable to postulate that pericytes might be involved in microvascular changes associated with COPD.

Ang2 functions as part of the Ang-Tie2 receptor axis that mediates blood vessel maturation and is mainly expressed during vascular remodelling by ECs and reportedly by perivascular cells as well [6]. Ang2 has been shown to be increased in the serum of COPD patients during exacerbations [70]. Ang2 facilitates the loosening of pericyte-endothelial interactions, leading to vessel destabilization, and, in conjunction with VEGF, promotes angiogenesis. Finally, the cell surface expression of CD146, a marker found on most pericytes, has been shown to be decreased in rat lung in an experimental model of emphysema and patients with COPD, leading to increased microvascular permeability, whilst soluble CD146 was increased in bronchoalveolar lavage fluid [71]. Although this study was performed to investigate the role of the endothelium in COPD, it cannot be ruled out that pericytes did not contribute to CD146 expression [71]. Taken together, although these studies do not provide direct evidence for a role of pericytes in COPD, they highlight areas that require further investigation.

\section{Pericytes in Asthma}

Allergic asthma is a respiratory disease driven by aeroallergen exposure and the subsequent allergen-driven immune response. The pathology of allergic asthma is characterized by airway remodelling, encompassing changes to the airway epithelium (excess mucus production and epithelial shedding) and subepithelial regions (angiogenesis, fibrosis and airway smooth muscle thickening) [72] . These structural changes to the airway wall and the pulmonary microvasculature ultimately lead to impaired lung function [73]. As with COPD, there are no currently published studies that have directly investigated the role of pericytes in allergic asthma. However, unpublished data from our laboratory suggest that, in a mouse model of house dust mite-induced allergic airway disease, pericytes are one of several cell types in the lung that contribute to airway smooth muscle thickening.

The vascular component in asthma has been more extensively studied compared with COPD. An increased number of vessels, increased vessel activity (vasodilation, leakage and leucocyte extravasation) and increased growth factor secretion have been observed in asthmatics compared with healthy control subjects [74]. Moreover, increased vascularity correlated with disease severity and likely contributed to the robust inflammatory response seen in severe asthma [74]. Interestingly, the capillaries of the lower airways are not usually fenestrated in the healthy lung [75], but this phenomenon has been observed in asthmatics [76]. Moreover, vascular remodelling, characterized by a thickening of the VSMC layer, EC proliferation and perivascular fibrosis, has been observed in the bronchi of mice subjected to respiratory ovalbumin (OVA) [77] and chronic house dust mite [78] exposure.

In addition to angiogenesis and vessel stability, VEGF mediates capillary fenestration and, as in certain subtypes of COPD, has been shown to be elevated in asthmatics and in IL-13-driven murine models of asthma [79]. Similarly, elevated levels of Ang2 in induced sputum correlate with asthma severity [80]. In a mouse model of allergic airway disease driven by OVA sensitization and challenge, Tie 2 expression has been observed in macrophages and epithelial cells, which did not express this Ang ligand under control conditions [80]. VEGF and Ang2 levels were also elevated in bronchoalveolar lavage fluid as well as whole lung tissue and expressed by VSMCs and MSCs [80]. Conversely, also in an OVA-driven model of allergic airway disease, Ang1 expression was decreased with respect to control mice and was thought to confer protection against airway inflammation [81]. Further research in this field will certainly clarify the role of Ang and vascular stability in asthma.

PDGF-BB/PDGFR- $\beta$ signalling is another important aspect of pericyte-endothelial interactions. Unpublished data from our group using a murine model of allergic asthma have shown that a disruption of this signalling pathway leads to pericyte mobilization away from the airway microvasculature and accumulation in the airway smooth muscle bundles; this process was found to significantly contribute to airway hyperreactivity in this model. Interestingly, the impact of PDGF-BB/PDGFR- $\beta$ signalling in mediating airway remodelling in allergic airway disease was shown in a study by Hirota et al. [82], in which the ectopic overexpression of PDGF-BB in the airway lumen of OVA-sensitized mice led to airway smooth muscle cell proliferation and increased airway hyperresponsiveness compared with mice exposed to the surrogate allergen OVA alone. Although the contribution of microvascular pericytes to these changes was not investigated, it has been shown that pericytes undergo chemotaxis towards a strong source of PDGF-BB [1]. Conversely, the inhibition of PDGF-BB/PDGFR- $\beta$ signalling by tyrosine kinase inhibitors (imatinib and masitinib) is currently being investigated as a means of reversing airway remodelling in animal models of 
asthma, and phase II studies are currently underway in human asthmatics (ClinicalTrials.gov identifier NCT01097694). This drug class has been quite extensively studied in cancer models; interestingly, the effect of imatinib intervention depends on the PDGF-BB expression level in tumour cells, since a PDGFR- $\beta$ blockade in high PDGF-BB-expressing tumours significantly inhibited tumour growth and metastases by increasing pericyte coverage and stabilizing the microvasculature, whereas in low PDGF-BB-expressing tumours, imatinib treatment reduced pericyte coverage and promoted tumour cell dissemination [83]. Tyrosine kinase inhibitors have shown therapeutic promise in cat [84] and mouse [85] models of allergic asthma, with reductions observed in airway inflammation and remodelling, leading to improved lung function. However, it must be emphasized that the compounds used in these studies on allergic asthma (masitinib and imatinib) have profound effects on the inflammatory response as well, greatly complicating the interpretation of results on structural changes and lung function. In any case, these studies highlight the importance of understanding the role of vascular mediators in asthma. As pericytes are involved in these signalling pathways and in vascular homoeostasis, it seems probable that they contribute to asthma pathobiology, and their contribution to these mechanisms requires further investigation.

These studies in animal models of kidney, liver and lung fibrosis point to a vital role of pericytes in myofibroblast activation, collagen deposition and microvascular remodelling observed in chronic lung disease (fig. 1c). However, there may be considerable differences in the mechanisms driving organ fibrosis in humans and those active in animals such as the mouse, most importantly in terms of the duration of the disease. Further studies into the mechanisms of the pericyte-to-myofibroblast transition and migration to fibrotic foci are certainly warranted, in addition to a confirmation of the importance of pericytes in human fibrotic disease.

\section{Pericytes in Pulmonary Hypertension}

Pulmonary hypertension is characterized by structural changes to the walls of pulmonary vessels, with prominent fibrosis of the vessel wall, an accumulation of a-SMA+ cells and inflammatory cell infiltration [86], ultimately leading to increased blood pressure in the pulmonary vasculature and heart failure. Currently, the molecular mechanisms responsible for these processes are

Pericytes in Chronic Lung Disease incompletely understood. In recent years, pericyte proliferation has been investigated as a causative factor of pulmonary hypertension.

Clinically, abnormal pericyte coverage of the pulmonary vasculature has been implicated in the pathogenesis of several forms of pulmonary hypertension, including congenital Adams-Oliver syndrome [87] and pulmonary capillary haemangiomatosis [88]. In particular, in the latter study, in situ hybridization and immunohistochemistry revealed a higher expression of PDGF-BB by type II pneumocytes and ECs as well as increased PDGFR- $\beta$ expression by pericytes and VSMCs associated with proliferating capillaries. This hypothesis was investigated in greater depth by Ricard et al. [19], using human material obtained during lung transplantations in pulmonary hypertension patients as well as from a chronic hypoxiadriven rodent model of pulmonary hypertension. An overabundance of pericytes was found to be associated with remodelled distal vessels in the lung in both the clinical samples and the rodent model; this was associated with an increased expression of FGF- 2 and IL- 6 by ECs, although no difference in PDGF-BB expression was observed [19]. The authors of this study concluded that pulmonary hypertension is driven by EC abnormalities that ultimately promote excess pericyte coverage, pulmonary smooth muscle hyperplasia and vascular remodelling. An increased coverage of the microvasculature with pericytes and VSMCs has also been observed in other animal models of pulmonary hypertension. Using a rat model of pulmonary hypertension driven by hyperoxia, Jones et al. [89] employed immunogold staining and high-resolution imaging to assess the PDGFR- $\beta / P D G F-B B$ pathway. PDGFR- $\beta$ was found to be highly overexpressed in perivascular cells in hypertensive lungs compared with control lungs; PDGF-BB was similarly overexpressed in the early stages of the disease [89]. It has been postulated by a number of authors that receptor tyrosine kinase inhibitors may be effective treatments for pulmonary hypertension as they have been shown to reduce pericyte density in solid tumour models [90]. However, the efficacy and safety of this intervention will need to be assessed in clinical trials on pulmonary hypertension patients.

\section{Future Directions}

It is clear that the vascular component of airway disease is of significance, and, despite being a key regulator of vascular mechanisms such as angiogenesis and vessel contractility, pericytes have been studied only recently in 
the context of IPF and pulmonary arterial hypertension. Moreover, the roles of this cell type in chronic lung diseases such as asthma and COPD are not completely understood at the present time. In addition to vascular homoeostasis, pericytes participate in inflammatory reac- tions and possess MSC-like progenitor capabilities, and are therefore likely to play multiple roles in lung pathobiology. A comprehensive understanding of the involvement of this cell type in chronic lung disorders may lead to the development of new therapeutic strategies.

\section{References}

-1 Armulik A, Abramsson A, Betsholtz C: Endothelial/pericyte interactions. Circ Res 2005; 97:512-523.

-2 Diaz-Flores L, Gutierrez R, Madrid JF, Varela $\mathrm{H}$, Valladares F, Acosta E, Martin-Vasallo P, Diaz-Flores L Jr: Pericytes. Morphofunction, interactions and pathology in a quiescent and activated mesenchymal cell niche. Histol Histopathol 2009;24:909-969.

-3 Feng J, Mantesso A, Sharpe PT: Perivascular cells as mesenchymal stem cells. Expert Opin Biol Ther 2010;10:1441-1451.

-4 Hall CN, Reynell C, Gesslein B, Hamilton NB, Mishra A, Sutherland BA, O'Farrell FM, Buchan AM, Lauritzen M, Attwell D: Capillary pericytes regulate cerebral blood flow in health and disease. Nature 2014;508:55-60.

5 Diaz-Flores L, Gutierrez R, Garcia MP, DiazFlores L Jr, Valladares F, Madrid JF: Ultrastructure of myopericytoma: a continuum of transitional phenotypes of myopericytes. Ultrastruct Pathol 2012;36:189-194.

6 Gaengel K, Genove G, Armulik A, Betsholtz $\mathrm{C}$ : Endothelial-mural cell signaling in vascular development and angiogenesis. Arterioscler Thromb Vasc Biol 2009;29:630-638.

7 Armulik A, Genove G, Betsholtz C: Pericytes: developmental, physiological, and pathological perspectives, problems, and promises. Dev Cell 2011;21:193-215.

$>8$ Bergers G, Song S: The role of pericytes in blood-vessel formation and maintenance. Neuro Oncol 2005;7:452-464

$>9$ Nehls V, Drenckhahn D: Heterogeneity of microvascular pericytes for smooth muscle type alpha-actin. J Cell Biol 1991;113:147-154.

-10 Corselli M, Crisan M, Murray IR, West CC, Scholes J, Codrea F, Khan N, Peault B: Identification of perivascular mesenchymal stro$\mathrm{mal} / \mathrm{stem}$ cells by flow cytometry. Cytometry A 2013;83:714-720.

-11 Crisan M, Yap S, Casteilla L, Chen CW, Corselli M, Park TS, Andriolo G, Sun B, Zheng B, Zhang L, et al: A perivascular origin for mesenchymal stem cells in multiple human organs. Cell Stem Cell 2008;3:301-313.

$>12$ Nees S, Weiss DR, Senftl A, Knott M, Forch S, Schnurr M, Weyrich P, Juchem G: Isolation, bulk cultivation, and characterization of coronary microvascular pericytes: the second most frequent myocardial cell type in vitro. Am J Physiol Heart Circ Physiol 2012; 302:H69-H84.
$>13$ Neng L, Zhang F, Kachelmeier A, Shi X: Endothelial cell, pericyte, and perivascular resident macrophage-type melanocyte interactions regulate cochlear intrastrial fluid-blood barrier permeability. J Assoc Res Otolaryngol 2013;14:175-185.

14 Tigges U, Welser-Alves JV, Boroujerdi A, Milner R: A novel and simple method for culturing pericytes from mouse brain. Microvasc Res 2012;84:74-80.

15 Lin SL, Kisseleva T, Brenner DA, Duffield JS: Pericytes and perivascular fibroblasts are the primary source of collagen-producing cells in obstructive fibrosis of the kidney. Am J Pathol 2008;173:1617-1627.

16 Birbrair A, Zhang T, Wang ZM, Messi ML, Enikolopov GN, Mintz A, Delbono O: Skeletal muscle pericyte subtypes differ in their differentiation potential. Stem Cell Res 2013;10: 67-84.

17 Mederacke I, Hsu CC, Troeger JS, Huebener P, Mu X, Dapito DH, Pradere JP, Schwabe RF: Fate tracing reveals hepatic stellate cells as dominant contributors to liver fibrosis independent of its aetiology. Nat Commun 2013; 4:2823.

18 Hung C, Linn G, Chow YH, Kobayashi A, Mittelsteadt K, Altemeier WA, Gharib SA, Schnapp LM, Duffield JS: Role of lung pericytes and resident fibroblasts in the pathogenesis of pulmonary fibrosis. Am J Respir Crit Care Med 2013;188:820-830.

19 Ricard N, Tu L, Le Hiress M, Huertas A, Phan C, Thuillet R, Sattler C, Seferian A, Fadel E, Montani D, et al: Increased pericyte coverage mediated by endothelial derived fibroblast growth factor- 2 and interleukin- 6 is a source of smooth muscle-like cells in pulmonary hypertension. Circulation 2014;129:1586-1597.

20 Rock JR, Barkauskas CE, Cronce MJ, Xue Y, Harris JR, Liang J, Noble PW, Hogan BL: Multiple stromal populations contribute to pulmonary fibrosis without evidence for epithelial to mesenchymal transition. Proc Natl Acad Sci USA 2011;108:E1475-E1483.

-21 Zehendner CM, Wedler HE, Luhmann HJ: A novel in vitro model to study pericytes in the neurovascular unit of the developing cortex. PLoS One 2013;8:e81637.

22 Friedenstein AJ, Piatetzky-Shapiro II, Petrakova KV: Osteogenesis in transplants of bone marrow cells. J Embryol Exp Morphol 1966; 16:381-390.
23 da Silva Meirelles L, Chagastelles PC, Nardi NB: Mesenchymal stem cells reside in virtually all post-natal organs and tissues. J Cell Sci 2006;119:2204-2213.

24 Dominici M, Le Blanc K, Mueller I, SlaperCortenbach I, Marini F, Krause D, Deans R, Keating A, Prockop D, Horwitz E: Minimal criteria for defining multipotent mesenchymal stromal cells. The International Society for Cellular Therapy position statement. Cytotherapy 2006;8:315-317.

25 Farrington-Rock C, Crofts NJ, Doherty MJ, Ashton BA, Griffin-Jones C, Canfield AE: Chondrogenic and adipogenic potential of microvascular pericytes. Circulation 2004; 110:2226-2232.

26 Spitzer TL, Rojas A, Zelenko Z, Aghajanova L, Erikson DW, Barragan F, Meyer M, Tamaresis JS, Hamilton AE, Irwin JC, Giudice LC: Perivascular human endometrial mesenchymal stem cells express pathways relevant to self-renewal, lineage specification, and functional phenotype. Biol Reprod 2012;86:58.

27 Li H, Usas A, Poddar M, Chen CW, Thompson S, Ahani B, Cummins J, Lavasani M, Huard J: Platelet-rich plasma promotes the proliferation of human muscle derived progenitor cells and maintains their stemness. PLoS One 2013;8:e64923.

28 Foronjy RF, Majka SM: The potential for resident lung mesenchymal stem cells to promote functional tissue regeneration: understanding microenvironmental cues. Cells 2012;1:874.

29 McQualter JL, Brouard N, Williams B, Baird BN, Sims-Lucas S, Yuen K, Nilsson SK, Simmons PJ, Bertoncello I: Endogenous fibroblastic progenitor cells in the adult mouse lung are highly enriched in the Sca-1 positive cell fraction. Stem Cells 2009;27:623-633.

30 Jun D, Garat C, West J, Thorn N, Chow K, Cleaver T, Sullivan T, Torchia EC, Childs C, Shade T, et al: The pathology of bleomycininduced fibrosis is associated with loss of resident lung mesenchymal stem cells that regulate effector T-cell proliferation. Stem Cells 2011;29:725-735.

31 Summer R, Fitzsimmons K, Dwyer D, Murphy J, Fine A: Isolation of an adult mouse lung mesenchymal progenitor cell population. Am J Respir Cell Mol Biol 2007;37:152-159. 
-32 Martin J, Helm K, Ruegg P, Varella-Garcia M, 45 Pieper C, MarekJJ, Unterberg M, Schwerdtle T, Burnham E, Majka S: Adult lung side population cells have mesenchymal stem cell potential. Cytotherapy 2008;10:140-151.

-33 Weibel ER: On pericytes, particularly their existence on lung capillaries. Microvasc Res 1974;8:218-235.

- 34 Postma DS, Reddel HK, ten Hacken NH, van den Berge M: Asthma and chronic obstructive pulmonary disease: similarities and differences. Clin Chest Med 2014;35:143-156.

- 35 Bringardner BD, Baran CP, Eubank TD, Marsh CB: The role of inflammation in the pathogenesis of idiopathic pulmonary fibrosis. Antioxid Redox Signal 2008;10:287-301.

-36 Nourshargh S, Hordijk PL, Sixt M: Breaching multiple barriers: leukocyte motility through venular walls and the interstitium. Nat Rev Mol Cell Biol 2010;11:366-378.

- 37 Wang S, Voisin MB, Larbi KY, Dangerfield J, Scheiermann C, Tran M, Maxwell PH, Sorokin L, Nourshargh S: Venular basement membranes contain specific matrix protein low expression regions that act as exit points for emigrating neutrophils. J Exp Med 2006; 203:1519-1532.

- 38 Voisin MB, Woodfin A, Nourshargh S: Monocytes and neutrophils exhibit both distinct and common mechanisms in penetrating the vascular basement membrane in vivo. Arterioscler Thromb Vasc Biol 2009;29: 1193-1199.

-39 Ayres-Sander CE, Lauridsen H, Maier CL, Sava P, Pober JS, Gonzalez AL: Transendothelial migration enables subsequent transmigration of neutrophils through underlying pericytes. PLoS One 2013;8:e60025.

40 Lauridsen HM, Pober JS, Gonzalez AL: A composite model of the human postcapillary venule for investigation of microvascular leukocyte recruitment. FASEB J 2014;28:11661180.

41 Stark K, Eckart A, Haidari S, Tirniceriu A, Lorenz M, von Bruhl ML, Gartner F, Khandoga AG, Legate KR, Pless R, et al: Capillary and arteriolar pericytes attract innate leukocytes exiting through venules and 'instruct' them with pattern-recognition and motility programs. Nat Immunol 2013;14:41-51.

42 Proebstl D, Voisin MB, Woodfin A, Whiteford J, D'Acquisto F, Jones GE, Rowe D, Nourshargh S: Pericytes support neutrophil subendothelial cell crawling and breaching of venular walls in vivo. J Exp Med 2012;209: 1219-1234.

43 Wang S, Cao C, Chen Z, Bankaitis V, Tzima E, Sheibani N, Burridge K: Pericytes regulate vascular basement membrane remodeling and govern neutrophil extravasation during inflammation. PLoS One 2012;7:e45499.

- 44 Hurtado-Alvarado G, Cabanas-Morales AM, Gomez-Gonzalez B: Pericytes: brain-immune interface modulators. Front Integr Neurosci 2014;7:80 Galla HJ: Brain capillary pericytes contribute to the immune defense in response to cytokines or LPS in vitro. Brain Res 2014;1550:1-8.

46 Tigges U, Boroujerdi A, Welser-Alves JV, Milner R: TNF- $\alpha$ promotes cerebral pericyte remodeling in vitro, via a switch from $\alpha 1$ to $\alpha 2$ integrins. J Neuroinflammation 2013;10:33.

47 Fuxe J, Tabruyn S, Colton K, Zaid H, Adams A, Baluk P, Lashnits E, Morisada T, Le T, O'Brien S, et al: Pericyte requirement for antileak action of angiopoietin-1 and vascular remodeling in sustained inflammation. Am J Pathol 2011;178:2897-2909.

48 Greenhalgh SN, Iredale JP, Henderson NC: Origins of fibrosis: pericytes take centre stage. F1000Prime Rep 2013;5:37.

49 Wynn TA: Cellular and molecular mechanisms of fibrosis. J Pathol 2008;214:199-210.

50 Rosenbloom J, Castro SV, Jimenez SA: Narrative review: fibrotic diseases - cellular and molecular mechanisms and novel therapies. Ann Intern Med 2010;152:159-166.

51 Steele MP, Schwartz DA: Molecular mechanisms in progressive idiopathic pulmonary fibrosis. Annu Rev Med 2013;64:265-276.

52 Moore BB, Lawson WE, Oury TD, Sisson TH, Raghavendran K, Hogaboam CM: Animal models of fibrotic lung disease. Am J Respir Cell Mol Biol 2013;49:167-179.

53 Wynn TA: Integrating mechanisms of pulmonary fibrosis. J Exp Med 2011;208:13391350 .

54 Greenhalgh SN, Conroy KP, Henderson NC: Healing scars: targeting pericytes to treat fibrosis. QJM 2014, Epub ahead of print.

-55 Dulauroy S, Di Carlo SE, Langa F, Eberl G, Peduto L: Lineage tracing and genetic ablation of $\mathrm{ADAM} 12^{+}$perivascular cells identify a major source of profibrotic cells during acute tissue injury. Nat Med 2012;18:1262-1270.

56 Humphreys BD, Lin SL, Kobayashi A, Hudson TE, Nowlin BT, Bonventre JV, Valerius MT, McMahon AP, Duffield JS: Fate tracing reveals the pericyte and not epithelial origin of myofibroblasts in kidney fibrosis. Am J Pathol 2010;176:85-97.

57 Kisseleva T, Cong M, Paik Y, Scholten D, Jiang C, Benner C, Iwaisako K, Moore-Morris T, Scott B, Tsukamoto H, et al: Myofibroblasts revert to an inactive phenotype during regression of liver fibrosis. Proc Natl Acad Sci USA 2012;109:9448-9453.

58 Karvonen HM, Lehtonen ST, Harju T, Sormunen RT, Lappi-Blanco E, Makinen JM, Laitakari K, Johnson S, Kaarteenaho RL: Myofibroblast expression in airways and alveoli is affected by smoking and COPD. Respir Res 2013;14:84.

59 Togo S, Holz O, Liu X, Sugiura H, Kamio K, Wang X, Kawasaki S, Ahn Y, Fredriksson K, Skold CM, et al: Lung fibroblast repair functions in patients with chronic obstructive pulmonary disease are altered by multiple mechanisms. Am J Respir Crit Care Med 2008;178: 248-260.
60 Hashimoto M, Tanaka H, Abe S: Quantitative analysis of bronchial wall vascularity in the medium and small airways of patients with asthma and COPD. Chest 2005;127:965-972.

61 Calabrese C, Bocchino V, Vatrella A, Marzo C, Guarino C, Mascitti S, Tranfa CM, Cazzola M, Micheli P, Caputi M, Marsico SA: Evidence of angiogenesis in bronchial biopsies of smokers with and without airway obstruction. Respir Med 2006;100:1415-1422.

62 Konigshoff M, Kneidinger N, Eickelberg O: TGF-beta signaling in COPD: deciphering genetic and cellular susceptibilities for future therapeutic regimen. Swiss Med Wkly 2009; 139:554-563

63 Barnes PJ: Mediators of chronic obstructive pulmonary disease. Pharmacol Rev 2004;56: 515-548.

64 Sieczkiewicz GJ, Herman IM: TGF- $\beta_{1}$ signaling controls retinal pericyte contractile protein expression. Microvasc Res 2003;66:190196.

65 Mendes ES, Campos MA, Wanner A: Airway blood flow reactivity in healthy smokers and in ex-smokers with or without COPD. Chest 2006;129:893-898.

66 Kranenburg AR, de Boer WI, Alagappan VK, Sterk PJ, Sharma HS: Enhanced bronchial expression of vascular endothelial growth factor and receptors (Flk-1 and Flt-1) in patients with chronic obstructive pulmonary disease. Thorax 2005;60:106-113.

-67 Kanazawa H, Asai K, Hirata K, Yoshikawa J: Possible effects of vascular endothelial growth factor in the pathogenesis of chronic obstructive pulmonary disease. Am J Med 2003;114: 354-358.

68 Kasahara Y, Tuder RM, Taraseviciene-Stewart L, Le Cras TD, Abman S, Hirth PK, Waltenberger J, Voelkel NF: Inhibition of VEGF receptors causes lung cell apoptosis and emphysema. J Clin Invest 2000;106: 1311-1319.

69 Darland DC, Massingham LJ, Smith SR, Piek E, Saint-Geniez M, D'Amore PA: Pericyte production of cell-associated VEGF is differentiation-dependent and is associated with endothelial survival. Dev Biol 2003;264:275288.

-70 Cho YJ, Ma JE, Yun EY, Kim YE, Kim HC, Lee JD, Hwang YS, Jeong YY: Serum angiopoietin-2 levels are elevated during acute exacerbations of COPD. Respirology 2011;16:284-290.

71 Kratzer A, Chu HW, Salys J, Moumen Z, Leberl M, Bowler R, Cool C, Zamora M, Taraseviciene-Stewart L: Endothelial cell adhesion molecule CD146: implications for its role in the pathogenesis of COPD. J Pathol 2013;230: 388-398.

72 Al-Muhsen S, Johnson JR, Hamid Q: Remodeling in asthma. J Allergy Clin Immunol 2011; 128:451-462, quiz 463-464.

73 Bousquet J, Vignola AM, Chanez P, Campbell AM, Bonsignore G, Michel FB: Airways remodelling in asthma: no doubt, no more? Int Arch Allergy Immunol 1995;107:211-214. 
74 Wilson JW, Hii S: The importance of the airway microvasculature in asthma. Curr Opin Allergy Clin Immunol 2006;6:51-55.

75 Zanini A, Chetta A, Imperatori AS, Spanevello A, Olivieri D: The role of the bronchial microvasculature in the airway remodelling in asthma and COPD. Respir Res 2010;11: 132.

76 McDonald DM: The ultrastructure and permeability of tracheobronchial blood vessels in health and disease. Eur Respir J Suppl 1990; 12:572s-585s.

77 Rydell-Törmänen K, Uller L, Erjefält JS: Allergic airway inflammation initiates longterm vascular remodeling of the pulmonary circulation. Int Arch Allergy Immunol 2009; 149:251-258.

78 Rydell-Törmänen K, Johnson JR, Fattouh R, Jordana M, Erjefält JS: Induction of vascular remodeling in the lung by chronic house dust mite exposure. Am J Respir Cell Mol Biol 2008;39:61-67.

79 Lee CG, Ma B, Takyar S, Ahangari F, Delacruz $\mathrm{C}, \mathrm{He} \mathrm{CH}$, Elias JA: Studies of vascular endothelial growth factor in asthma and chronic obstructive pulmonary disease. Proc Am Thorac Soc 2011;8:512-515.

80 Makinde TO, Agrawal DK: Increased expression of angiopoietins and Tie2 in the lungs of chronic asthmatic mice. Am J Respir Cell Mol Biol 2011;44:384-393.
81 Simoes DC, Vassilakopoulos T, Toumpanakis D, Petrochilou K, Roussos C, Papapetropoulos A: Angiopoietin-1 protects against airway inflammation and hyperreactivity in asthma. Am J Respir Crit Care Med 2008;177:13141321.

82 Hirota JA, Ask K, Farkas L, Smith JA, Ellis R, Rodriguez-Lecompte JC, Kolb M, Inman MD: In vivo role of platelet-derived growth factor$\mathrm{BB}$ in airway smooth muscle proliferation in mouse lung. Am J Respir Cell Mol Biol 2011; 45:566-572.

83 Hosaka K, Yang Y, Seki T, Nakamura M, Andersson P, Rouhi P, Yang X, Jensen L, Lim S, Feng N, et al: Tumour PDGF-BB expression levels determine dual effects of anti-PDGF drugs on vascular remodelling and metastasis. Nat Commun 2013;4:2129.

84 Lee-Fowler TM, Guntur V, Dodam J, Cohn LA, DeClue AE, Reinero CR: The tyrosine kinase inhibitor masitinib blunts airway inflammation and improves associated lung mechanics in a feline model of chronic allergic asthma. Int Arch Allergy Immunol 2012;158: 369-374.
85 Rhee CK, Kim JW, Park CK, Kim JS, Kang JY, Kim SJ, Kim SC, Kwon SS, Kim YK, Park SH, Lee SY: Effect of imatinib on airway smooth muscle thickening in a murine model of chronic asthma. Int Arch Allergy Immunol 2011;155:243-251.

86 Yeager ME, Frid MG, Stenmark KR: Progenitor cells in pulmonary vascular remodeling. Pulm Circ 2011;1:3-16.

87 Patel MS, Taylor GP, Bharya S, Al-Sanna’a N, Adatia I, Chitayat D, Suzanne Lewis ME, Human DG: Abnormal pericyte recruitment as a cause for pulmonary hypertension in AdamsOliver syndrome. Am J Med Genet A 2004; 129A:294-299.

88 Assaad AM, Kawut SM, Arcasoy SM, Rosenzweig EB, Wilt JS, Sonett JR, Borczuk AC: Platelet-derived growth factor is increased in pulmonary capillary hemangiomatosis. Chest 2007;131:850-855

89 Jones R, Capen D, Jacobson M: PDGF and microvessel wall remodeling in adult lung: imaging PDGF-R $\beta$ and PDGF-BB molecules in progenitor smooth muscle cells developing in pulmonary hypertension. Ultrastruct Pathol 2006;30:267-281.

90 Pietras K, Pahler J, Bergers G, Hanahan D: Functions of paracrine PDGF signaling in the proangiogenic tumor stroma revealed by pharmacological targeting. PLoS Med 2008; 5:e19. 